\title{
Electric linear dichroism transients of bent DNA fragments
}

Juan P. Umazano, and Jorge A. Bertolotto

Citation: The Journal of Chemical Physics 138, 095102 (2013); doi: 10.1063/1.4793984

View online: https://doi.org/10.1063/1.4793984

View Table of Contents: http://aip.scitation.org/toc/jcp/138/9

Published by the American Institute of Physics

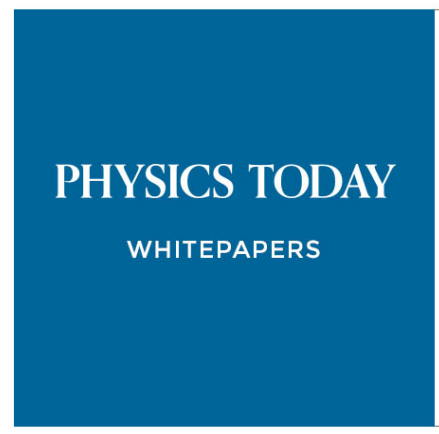




\title{
Electric linear dichroism transients of bent DNA fragments
}

\author{
Juan P. Umazano ${ }^{1,2, a)}$ and Jorge A. Bertolotto ${ }^{1}$ \\ ${ }^{1}$ Facultad de Ciencias Exactas y Naturales, Universidad Nacional de La Pampa, Santa Rosa, \\ L6300CLB, Argentina \\ ${ }^{2}$ Consejo Nacional de Investigaciones Científicas y Técnicas (CONICET), Buenos Aires, C1033AAJ, Argentina
}

(Received 28 December 2012; accepted 17 February 2013; published online 6 March 2013)

\begin{abstract}
We study the effect of translational-rotational hydrodynamic coupling on the transient electric linear dichroism of DNA fragments in aqueous solution. As opposed to previous theoretical works, where analytic solutions valid in the limit of low electric field were reported, we present here a numerical approach which allows to obtain numerical results valid independently from the applied electric field strength. Numerical procedures here used are an extension to the transient-state of those developed in a previous work for the study of the problem in the steady-state. The molecular orientational processes induced by an electric field is characterized with statistical arguments solving the FokkerPlanck equation by means of the finite difference method to know the orientational distribution function of molecules. @ 2013 American Institute of Physics. [http://dx.doi.org/10.1063/1.4793984]
\end{abstract}

\section{INTRODUCTION}

Electro-optical techniques are a useful tool to study physical properties of macromolecules in solution. Experimental data of electric birefringence and dichroism depend on optic, electric, and hydrodynamic properties of macromolecules. For particles with simple geometrical shapes, as cylinders or ellipsoids, the analysis of experimental data is relatively simple. However, for asymmetrical shaped particles the analysis results more difficult due to the hydrodynamic coupling between their translational and rotational movements. ${ }^{1} \mathrm{Bi}-$ ological macromolecules, like DNA fragments with length chain greater than persistence length, are an example of nonsymmetrical particles.

An analytic study of transient electric birefringence for polar and polarizable molecules with arbitrary shape in strong electric fields has been reported by Kalmykov, ${ }^{2}$ but that work does not include the hydrodynamic coupling of molecules. Nowadays, the influence of hydrodynamic coupling on the electro-optical effects (electric birefringence and dichroism) of DNA fragments has been studied through Brownian dynamic simulation ${ }^{3-5}$ and theoretical methods. ${ }^{6-15}$ Theoretical analytic results have been reported only for the limit of low electric field strength because the study of the orientational process induced by electric field has mathematical difficulties. Simulation studies early made are applied for arbitrary electric field strength but they have a disadvantage; they require computer simulations that are too extensive. In the past, applications of theoretical methods in combination with numerical techniques allowed us to remove the low electric field condition for the theoretical study of electric dichroism of DNA fragments in the steady-state. ${ }^{14}$ In the present work, we propose to extend that calculation to study the transient electric dichroism of DNA fragments. We use a rigid bent rod molecule (BRM) model to describe the particles in solution. Transient electro-optical effect is calculated using the orienta-

\footnotetext{
a)pumazano@exactas.unlpam.edu.ar.
}

tional distribution function obtained numerically solving the Fokker-Planck equation dependent on time. We specially analyze the effect of translational-rotational hydrodynamic coupling on the transient electric dichroism. This study includes both growth and decay of electro-optical effect when the electric field is set-on and set-off, respectively. The results are analyzed for electric fields with different strengths.

\section{THEORETICAL BACKGROUND}

The theoretical background here used has been thoroughly described in previous works. ${ }^{12-14}$ Therefore, we will develop this section only in a general way.

\section{A. Molecular model}

Each DNA fragment is modelled as a rigid BRM geometrically characterized by their arc length $S_{0}$, curvature radius $R$, and bending angle $2 \gamma_{0}$ (Figure 1). The BRM is put on the plane $X^{\prime} Z^{\prime}$ of the body coordinate system $O^{\prime} X^{\prime} Y^{\prime} Z^{\prime}$ which origin $O^{\prime}$ coincides with the BRM center of mass. The molecule electric properties are its electric charge $q$ and its electric polarizability tensor $\left[\alpha^{E}\right]$ with principal elements $\alpha_{x^{\prime} x^{\prime}}^{E}, \alpha_{y^{\prime} y^{\prime}}^{E}$, and $\alpha_{z^{\prime} z^{\prime}}^{E}$. The hydrodynamic properties of BRM are given by its rotational diffusion tensor $[R]$ with principal elements $R_{x^{\prime} x^{\prime}}$, $R_{y^{\prime} y^{\prime}}$, and $R_{z^{\prime} z^{\prime}}$, and the translation-rotation coupling diffusion tensor $[P]$ with nonzero elements $P_{y^{\prime} z^{\prime}}$ and $P_{z^{\prime} y^{\prime}}$. The optical properties are represented by the transition moment of each BRM chromophore group. The position and orientation of BRM with respect to the laboratory coordinate system $O X Y Z$ are given by the coordinates $x, y, z$ of the $O^{\prime}$ origin of the body coordinate system and the Euler angles $\phi, \theta$, and $\psi$.

\section{B. Reduced electric linear dichroism}

We considered a diluted solution of DNA fragments in presence of a static electric field whose direction match 


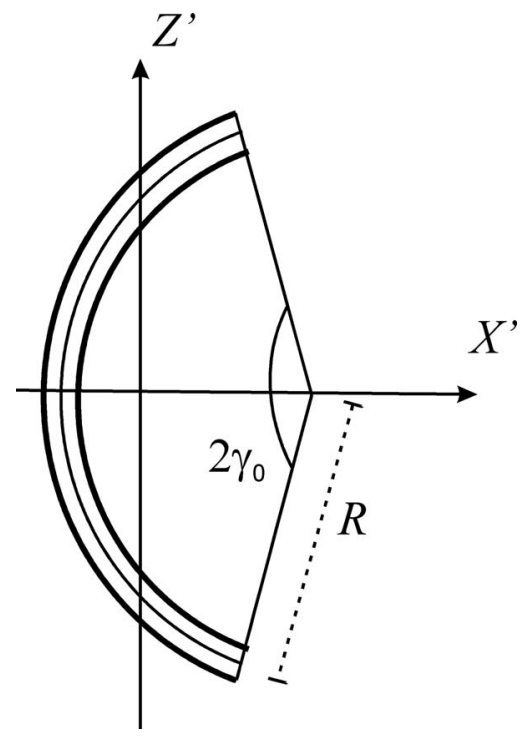

FIG. 1. Bent rod molecule (BRM) in the body coordinate system.

with the $Z$ axis direction of the laboratory coordinate system. The reduced electric linear dichroism (RELD) is defined as

$$
\frac{\Delta A}{A}=\frac{A_{/ /}-A_{\perp}}{A},
$$

where $A_{/ /}$and $A_{\perp}$ are the absorbances for linearly polarized incident light according to the parallel and perpendicular directions of the applied electric field, and $A$ is the absorbance of the solution for randomly oriented molecules.

In a previous work $^{14}$ an expression for the RELD in the steady-state of a solution of DNA fragments described with the BRM model was deduced. This expression can be immediately generalized for transient RELD as follows

$$
\begin{aligned}
\frac{\Delta A}{A}= & \frac{3 \pi}{16 \gamma_{o}} \int_{0}^{2 \pi} \int_{0}^{\pi}\left[\left(-\gamma_{o}-3 A_{o}\right)(1+3 \cos 2 \theta)\right. \\
& \left.-6\left(-\gamma_{o}+A_{o}\right) \cos 2 \psi \sin ^{2} \theta\right] f(\theta, \psi, t) \sin \theta d \theta d \psi,
\end{aligned}
$$

where $A_{o}=\cos \gamma_{o} \sin \gamma_{o}$ and $f(\theta, \psi, t)$ is the orientational distribution function. This function describes the probability of finding a molecule with an orientation between the angles $(\theta, \psi)$ and $(\theta+d \theta, \psi+d \psi)$ at time $t$ with respect to the laboratory coordinate system. The orientational distribution function is not dependent of angle $\phi$ due to the fact that the electric field induces a cylindrical symmetry in the solution.

\section{Orientational process}

We denominate $f(\boldsymbol{\Omega}, t)$ to the probability density of finding a molecule with coordinates between $\boldsymbol{\Omega}=(x, y, z, \phi, \theta$, $\psi)$ and $\boldsymbol{\Omega}+d \boldsymbol{\Omega}$ at instant $t$ with respect to the laboratory coordinate system. The probability density $f(\boldsymbol{\Omega}, t)$ is the solution of the Fokker-Planck equation given by

$$
\frac{\partial f(\boldsymbol{\Omega}, t)}{\partial t}=-\frac{1}{\sqrt{g}} \vec{\nabla}_{\boldsymbol{\Omega}} \cdot[\sqrt{g} \mathbf{J}(\boldsymbol{\Omega}, t)],
$$

where operator $\vec{\nabla}_{\boldsymbol{\Omega}}$ is the vector $(\partial / \partial x, \partial / \partial y, \partial / \partial z, \partial / \partial \phi, \partial / \partial \theta$, $\partial / \partial \psi), g=\sin ^{2} \theta$ is the determinant of the metric tensor, and $\mathbf{J}(\boldsymbol{\Omega}, t)$ is the probability current which depends on the drift and diffusion moments $\mathbf{M}_{1}$ and $\left[M_{2}\right]$, respectively, in the following way

$$
\mathbf{J}(\boldsymbol{\Omega}, t)=\mathbf{M}_{1} f(\boldsymbol{\Omega}, t)-\frac{1}{2}\left[M_{2}\right] \vec{\nabla}_{\boldsymbol{\Omega}} f(\boldsymbol{\Omega}, t) .
$$

We suppose that the probability density is independent of the coordinate positions and also of the $\phi$ angle. In this way, $f(\boldsymbol{\Omega}, t)$ becomes the orientational distribution function $f(\theta, \psi$, $t$ ). Under these suppositions Eq. (3) was developed in previous works $^{12,14}$ and results in the following differential equation

$$
\left(Q_{\theta \theta} \frac{\partial^{2}}{\partial \theta^{2}}+Q_{\psi \psi} \frac{\partial^{2}}{\partial \psi^{2}}+Q_{\psi \theta} \frac{\partial^{2}}{\partial \psi \partial \theta}+Q_{\theta} \frac{\partial}{\partial \theta}+Q_{\psi} \frac{\partial}{\partial \psi}+Q_{00}\right) f(\theta, \psi, t)=\frac{\partial f(\theta, \psi, t)}{\partial t},
$$

where

$$
\begin{aligned}
Q_{\theta \theta}= & \left(R_{x^{\prime} x^{\prime}} \cos ^{2} \psi+R_{y^{\prime} y^{\prime}} \sin ^{2} \psi\right), \\
Q_{\psi \psi}= & \left(R_{x^{\prime} x^{\prime}} \sin ^{2} \psi \cot ^{2} \theta+R_{y^{\prime} y^{\prime}} \cos ^{2} \psi \cot ^{2} \theta+R_{z^{\prime} z^{\prime}}\right), \\
Q_{\psi \theta}= & 2\left(R_{y^{\prime} y^{\prime}}-R_{x^{\prime} x^{\prime}}\right) \sin \psi \cos \psi \cot \theta, \\
Q_{\theta}= & \cot \theta\left(R_{x^{\prime} x^{\prime}} \sin ^{2} \psi+R_{y^{\prime} y^{\prime}} \cos ^{2} \psi\right)-\frac{E^{2}}{k_{B} T} \cos \theta \sin \theta\left[\left(\alpha_{y^{\prime} y^{\prime}}^{E}-\alpha_{z^{\prime} z^{\prime}}^{E}\right) R_{x^{\prime} x^{\prime}} \cos ^{2} \psi\right. \\
& \left.-\left(\alpha_{z^{\prime} z^{\prime}}^{E}-\alpha_{x^{\prime} x^{\prime}}^{E}\right) R_{y^{\prime} y^{\prime}} \sin ^{2} \psi\right]+\frac{q E}{k_{B} T} P_{y^{\prime} z^{\prime}} \cos \theta \sin \psi, \\
Q_{\psi}= & \frac{\cos \psi}{\sin ^{2} \theta}\left\{\frac{E^{2}}{k_{B} T}\left[R_{z^{\prime} z^{\prime}}\left(\alpha_{y^{\prime} y^{\prime}}^{E}-\alpha_{x^{\prime} x^{\prime}}\right) \sin ^{2} \theta+R_{y^{\prime} y^{\prime}}\left(\alpha_{z^{\prime} z^{\prime}}^{E}-a_{x^{\prime} x^{\prime}}^{E}\right) \cos ^{2} \theta+R_{x^{\prime} x^{\prime}}\left(\alpha_{y^{\prime} y^{\prime}}^{E}-\alpha_{z^{\prime} z^{\prime}}^{E}\right) \cos ^{2} \theta\right]\right. \\
& \left.\times \sin ^{2} \theta \sin \psi+\frac{E q}{k_{B} T} \sin \theta\left(P_{y^{\prime} z^{\prime}} \cos ^{2} \theta-P_{z^{\prime} y^{\prime}} \sin ^{2} \theta\right)+\left(R_{x^{\prime} x^{\prime}}-R_{y^{\prime} y^{\prime}}\right) \sin \psi\left(\cos ^{2} \theta+1\right)\right\},
\end{aligned}
$$




$$
\begin{aligned}
Q_{00}= & -\frac{1}{\sin \theta}\left\{\frac{q E}{k_{B} T}\left(P_{y^{\prime} z^{\prime}}-P_{z^{\prime} y^{\prime}}\right) \sin \psi \sin ^{2} \theta+\frac{E^{2}}{k_{B} T} R_{z^{\prime} z^{\prime}}\left(\alpha_{x^{\prime} x^{\prime}}^{E}-\alpha_{y^{\prime} y^{\prime}}^{E}\right) \sin ^{3} \theta\left(2 \cos ^{2} \psi-1\right)\right. \\
& \left.+\frac{E^{2}}{k_{B} T} \sin \theta\left[R_{x^{\prime} x^{\prime}}\left(\alpha_{y^{\prime} y^{\prime}}^{E}-\alpha_{z^{\prime} z^{\prime}}^{E}\right)\left(\cos ^{2} \theta-\sin ^{2} \theta \cos ^{2} \psi\right) R_{y^{\prime} y^{\prime}}\left(\alpha_{z^{\prime} z^{\prime}}^{E}-\alpha_{x^{\prime} x^{\prime}}^{E}\right)+\left(\sin ^{2} \theta \sin ^{2} \psi-\cos ^{2} \theta\right)\right]\right\} .
\end{aligned}
$$

We express the orientational distribution function in the following way

$$
f(\theta, \psi, t)=f^{0}+f^{E}(\theta, \psi, t),
$$

where $f^{0}$ is the orientational distribution function before applying the electric field and $f^{E}(\theta, \psi, t)$ represents the modifications at instant $t$ after applying the electric field. Let us note that $f^{0}$ is a constant because before applying the electric field all orientations are equally probable. Replacing Eq. (6) in Eq. (5), we obtain

$$
\hat{Q} f^{E}(\theta, \psi, t)=-Q_{00} f^{0}+\frac{\partial f^{E}(\theta, \psi, t)}{\partial t},
$$

where $\widehat{Q}=Q_{\theta \theta} \partial^{2} / \partial \theta^{2}++Q_{\psi \psi} \partial^{2} / \partial \psi^{2}+Q_{\psi \theta} \partial^{2} / \partial \psi \partial \theta+$ $Q_{\theta} \partial / \partial \theta+Q_{\psi} \partial / \partial \psi+Q_{00}$.

The orientational distribution function is obtained solving Eq. (7) to know $f^{E}(\theta, \psi, t)$ and replacing it in Eq. (6).

\section{NUMERICAL PROCEDURE TO CALCULATE THE RELD}

The RELD is numerically calculated by means of Eq. (2). For this, we need to determine previously the orientational distribution function $f(\theta, \psi, t)$. To this end, we solve the differential equation given by Eq. (7) using a finite difference method (FDM) $)^{16}$ as is described in a previous work. ${ }^{17} \mathrm{Ba}-$ sically, the mentioned numerical procedure consists first in making a grid of discrete points $\theta_{l}, \psi_{m}$ on the domain $0 \leq \theta$ $\leq \pi$ and $0 \leq \psi \leq 2 \pi$, with mesh sizes $\Delta \theta=\pi /\left(n_{\theta}-1\right)$ and $\Delta \psi=2 \pi /\left(n_{\psi}-1\right)$, being $n_{\theta}$ and $n_{\psi}$ the number of points in the $\theta$ and $\psi$ directions, respectively. Second, we approximate the angular and temporal derivates in the differential equation using Taylor series. Finally, we generate an equation system which solution is the set of values that are assumed by $f^{E}(\theta$, $\psi, t_{k}$ ) in the grid points at instant $t_{k}$ after applying the electric field. The equation system, which is obtained from the discretization of the differential equation and the boundary conditions, is generated by the following relations ${ }^{17}$

$$
\begin{gathered}
H_{1} f_{l+1, m, k}^{E}+\left(H_{2}-\Delta t^{-1} f_{l, m, k-1}^{E}\right) f_{l, m, k}^{E}+H_{3} f_{l-1, m, k}^{E}+H_{4} f_{l, m+1, k}^{E}+H_{5} f_{l, m-1, k}^{E} \\
+H_{6} f_{l+1, m+1, k}^{E}+H_{7} f_{l-1, m-1, k}^{E}=H_{8} f^{0}-\Delta t^{-1} f_{l, m, k-1}^{E} \quad l=1,2, \ldots, n_{\theta}-2 \\
m=0,1, \ldots, n_{\psi}-1, \\
f_{l, 0, k}^{E}-f_{l, n_{\psi}-1, k}^{E}=0 \quad l=0,1,2, \ldots, n_{\theta}-1, \\
\frac{1}{4 \Delta \psi}\left[R_{y^{\prime} y^{\prime}}+R_{z^{\prime} z^{\prime}}+\left(R_{y^{\prime} y^{\prime}}-R_{z^{\prime} z^{\prime}}\right) \cos (2 \theta)\right]\left(f_{l,-1, k}^{E}-f_{l, 1, k}^{E}-f_{l, n_{\psi}-2, k}^{E}+f_{l, n_{\psi}, k}^{E}\right) \\
+\frac{q E}{k_{B} T}\left[P_{z^{\prime} y^{\prime}} \sin ^{2} \theta-P_{y^{\prime} z^{\prime}} \cos { }^{2} \theta\right] \sin \theta\left(f_{l, 0, k}^{E}-f_{l, n_{\psi}-1, k}^{E}\right)=0 \quad l=0,1, \ldots, n_{\theta}-1, \\
\frac{1}{\Delta \theta}\left[\left(R_{x^{\prime} x^{\prime}}+R_{y^{\prime} y^{\prime}}\right)+\left(R_{x^{\prime} x^{\prime}}-R_{y^{\prime} y^{\prime}}\right) \cos (2 \psi)\right] f_{1, m, k}^{E}-\left\{\frac{1}{\Delta \theta}\left[\left(R_{x^{\prime} x^{\prime}}+R_{y^{\prime} y^{\prime}}\right)+\left(R_{x^{\prime} x^{\prime}}-R_{y^{\prime} y^{\prime}}\right) \cos (2 \psi)\right]\right. \\
\left.-\frac{2 q E}{k_{B} T} P_{y^{\prime} z^{\prime}} \sin \psi\right\} f_{0, m, k}^{E}=-\frac{2 q E}{k_{B} T} P_{y^{\prime} z^{\prime}} \sin \psi \quad m=0,1,2, \ldots, n_{\psi}-1, \\
-\frac{1}{\Delta \theta}\left[\left(R_{x^{\prime} x^{\prime}}+R_{y^{\prime} y^{\prime}}\right)+\left(R_{x^{\prime} x^{\prime}}-R_{y^{\prime} y^{\prime}}\right) \cos (2 \psi)\right] f_{n_{\theta}-2, m, k}^{E}+\left\{\frac{1}{\Delta \theta}\left[\left(R_{x^{\prime} x^{\prime}}+R_{y^{\prime} y^{\prime}}\right)+\left(R_{x^{\prime} x^{\prime}}-R_{y^{\prime} y^{\prime}}\right) \cos (2 \psi)\right]\right. \\
\left.\frac{2 q E}{k_{B} T} P_{y^{\prime} z^{\prime}} \sin \psi\right\} f_{n_{\theta}-1, m, k}^{E}=\frac{2 q E}{k_{B} T} P_{y^{\prime} z^{\prime}} \sin \psi \quad m=0,1,2, \ldots, n_{\psi}-1 .
\end{gathered}
$$


In the above equations, the subscripts $p, q, r$, with $p=l$, $l \pm 1, q=m, m \pm 1$ and $r=k-1, k$, mean that the function $f^{E}$ must be evaluated at the point $\theta_{p}, \psi_{q}$ and at the instant $t_{r}$; $\Delta t=t_{k}-t_{k-1}$ is the interval of time discretization, and $H_{i}$ are coefficients given by

$$
\begin{aligned}
& H_{1}=\frac{Q_{\theta \theta}}{\Delta \theta^{2}}-\frac{Q_{\psi \theta}}{2 \Delta \theta \Delta \psi}+\left.\frac{Q_{\theta}}{2 \Delta \theta}\right|_{l, m}, \\
& H_{2}=-\frac{2 Q_{\theta \theta}}{\Delta \theta^{2}}-\frac{2 Q_{\psi \psi}}{\Delta \psi^{2}}+\frac{Q_{\psi \theta}}{\Delta \theta \Delta \psi}+\left.Q_{00}\right|_{l, m}, \\
& H_{3}=\frac{Q_{\theta \theta}}{\Delta \theta^{2}}-\frac{Q_{\psi \theta}}{2 \Delta \theta \Delta \psi}-\left.\frac{Q_{\theta}}{2 \Delta \theta}\right|_{l, m}, \\
& H_{4}=\frac{Q_{\psi \psi}}{\Delta \psi^{2}}-\frac{Q_{\psi \theta}}{2 \Delta \theta \Delta \psi}+\left.\frac{Q_{\psi}}{2 \Delta \psi}\right|_{l, m}, \\
& H_{5}=\frac{Q_{\psi \psi}}{\Delta \psi \psi^{2}}-\frac{Q_{\psi \theta}}{2 \Delta \theta \Delta \psi}-\left.\frac{Q_{\psi}}{2 \Delta \psi}\right|_{l, m}, \\
& H_{6}=\left.\frac{Q_{\psi \theta}}{2 \Delta \theta \Delta \psi}\right|_{l, m}, \\
& H_{7}=H_{6} \\
& H_{8}=-\left.Q_{00}\right|_{l, m} .
\end{aligned}
$$

The equation system given by Eqs. (8)-(12) should be iteratively solved. From the first iteration $(k=1)$ the values of $f^{E}\left(\theta, \psi, t_{1}\right)$ are determined using the initial condition $f^{E}(\theta$, $\left.\psi, t_{0}\right)=f^{0}$, where $t_{0}=0$ is the instant at which the electric field is applied. As $f^{0}$ is an arbitrary constant, we choose $f^{0}=1$ to make the calculations. In the second iteration, the values of $f^{E}\left(\theta, \psi, t_{2}\right)$ are determined using the values of $f^{E}(\theta$, $\left.\psi, t_{1}\right)$ obtained in the first iteration. In this way, repeating this procedure, the values of $f^{E}$ at instant $t_{k}$ are determined solving the equation system given by Eqs. (8)-(12) on the basis of the values of $f^{E}$ at instant $t_{k-1}$.

At each instant $t_{k}$ the orientational distribution function $f\left(\theta, \psi, t_{k}\right)$ is calculated using Eq. (6) and the values of $f^{E}(\theta$, $\left.\psi, t_{k}\right)$ obtained for this iteration. Finally, the function $f(\theta, \psi$, $t_{k}$ ) is redefined in order to satisfy the following normalization condition

$$
f\left(\theta, \psi, t_{k}\right)=\frac{f^{0}+f^{E}\left(\theta, \psi, t_{k}\right)}{2 \pi \int_{0}^{2 \pi} \int_{0}^{\pi}\left(f^{0}+f^{E}\right) \sin \theta d \theta d \psi} .
$$

Let us note that the above procedure is useful to study the growth of RELD after electric field was applied, and also the decay of RELD after removing electric field. For the latter, we merely need to make $E=0$ in the equations and continue the iterative process.

Finally, transient REDL can be calculate by integration of Eq. (2) for different instants $t_{k}$.

\section{RESULTS AND DISCUSSION}

All calculations were made using a grid with $n_{\theta}=n_{\psi}$ $=91$ points and an interval of time discretization $\Delta t=0.1$ $\mu$ s to solve the Fokker-Planck equation, like it is described in Sec. III.

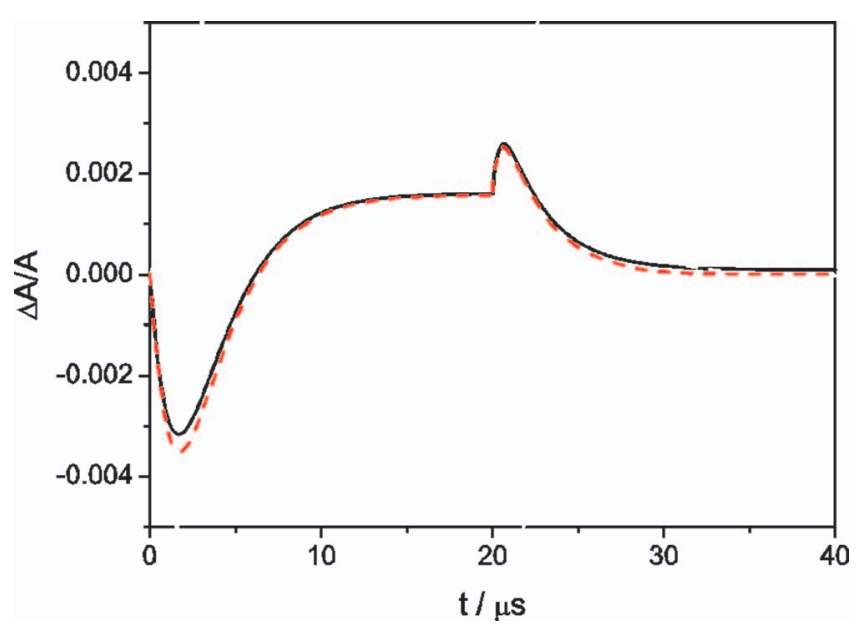

FIG. 2. RELD versus time for BRM solution with the parameters described in the text. Solid black line: values obtained solving Fokker-Planck equation with MDF, Dashed red line: values obtained with approximate analytic results reported by Bertolotto et al. ${ }^{13}$ Electric field with strength $2 \mathrm{kV} / \mathrm{cm}$ is set-on at $t=0$ and set-off at $t=20 \mu \mathrm{s}$.

We calculated the transient RELD according to theoretical and numerical procedures described in this work, for a BRM model with a bending angle of $116^{\circ}$ and arc length of $600 \AA$. The molecular parameters here used are the same implemented by Bertolotto et al. ${ }^{13}$ in a previous work. The principal elements of electric polarizability tensor are $\alpha_{x^{\prime} x^{\prime}}^{E}=7.311 \times 10^{-33} \mathrm{Fm}^{2}, \alpha_{y^{\prime} y^{\prime}}^{E}=5.341 \times 10^{-33} \mathrm{Fm}^{2}$, and $\alpha_{z^{\prime} z^{\prime}}^{E}=2.397 \times 10^{-32} \mathrm{Fm}^{2}$. The elements of the hydrodynamic tensors are $R_{x^{\prime} x^{\prime}}=63640 \mathrm{~s}^{-1}, R_{y^{\prime} y^{\prime}}=67960 \mathrm{~s}^{-1}$, and $R_{z^{\prime} z^{\prime}}=641800 \mathrm{~s}^{-1}, P_{y^{\prime} z^{\prime}}=2.847 \times 10^{-4} \mathrm{~m} / \mathrm{s}$ and $P_{z^{\prime} y^{\prime}}$ $=-4.285 \times 10^{-4} \mathrm{~m} / \mathrm{s}$. Electric charge of BRM is $q=0.16 Q$, where $Q$ is the DNA fragment charge when all the phosphate groups are ionized. Temperature $T$ is set on $293 \mathrm{~K}$. RELD is always calculated from $t=0$ to $t=40 \mu \mathrm{s}$ and the electric rectangular pulse is set-on at $t=0$ and set-off at $t=20 \mu \mathrm{s}$.

Figure 2 shows the RELD curves versus time obtained for BRM with bending angle $116^{\circ}$ and an electric field strength $\mathrm{E}$ $=2 \mathrm{kV} / \mathrm{cm}$. Figure 2 also shows the RELD curves calculated through approximate analytic results reported by Bertolotto et $a l .{ }^{13}$ valid in the limit of low electric field strength (up to $2 \mathrm{kV} / \mathrm{cm}$, approximately). Both calculations are in satisfactory agreement. During the first moments after the electric field is applied the RELD in Figure 2 is negative. Then, RELD becomes positive and grows until achieving a steady-state value. When electric field is set-off an overshoot occurs and then RELD decays to zero. Behaviour above described is characteristic of BRM that are oriented by two mechanisms. One of them is due to the interaction between the induce dipole moment and the electric field and the other is owned to the hydrodynamic coupling between the translational and rotational movements.

Figure 3 shows the RELD curves versus time obtained for BRM with bending angle $116^{\circ}$ and an electric field strength $\mathrm{E}=2 \mathrm{kV} / \mathrm{cm}$ calculated considering each orientation mechanism individually. The orientation by polarization produces a RELD signal always negative. On the contrary, the orientation owned to hydrodynamic coupling corresponds a RELD 


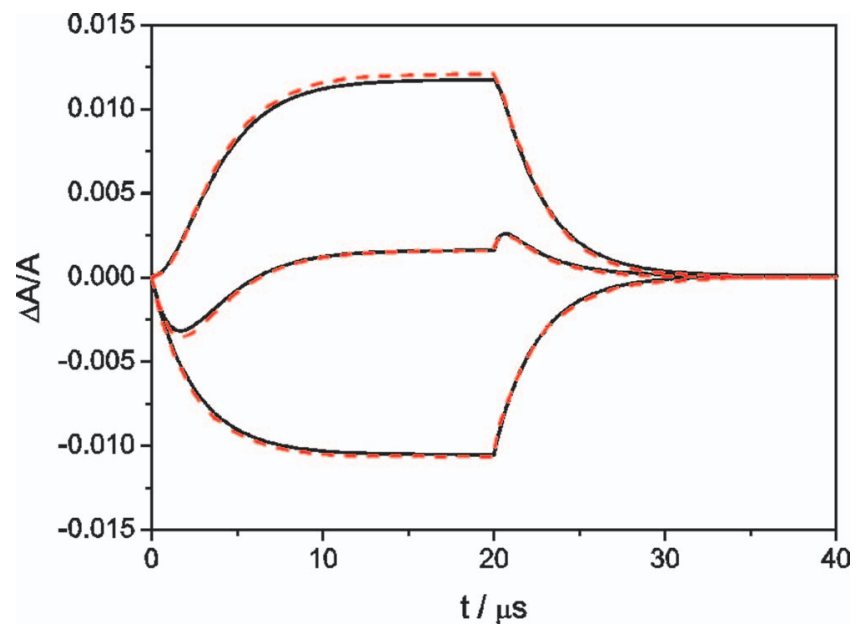

FIG. 3. Individual contributions to RELD curve of Figure 2. Solid black line: values obtained solving Fokker-Planck equation with MDF, Dashed red line: values obtained with approximate analytic results reported by Bertolotto et al. ${ }^{13}$ Always positive curves represent the contribution owned to hydrodynamic coupling and always negative curves those owned to polarization. The middle curves correspond to both contributions together.

signal always positive. The combination of both contributions results in the curve of Figure 2. In Figure 3 we can see that curves calculated with the MDF and those analytic curves reported by Bertolotto et al. ${ }^{13}$ are in agreement.

Figure 4 shows the different contributions to RELD for an arc with the same parameters used in Figure 3 in presence of an electric field with strength equal to $60 \mathrm{kV} / \mathrm{cm}$. The contribution of the hydrodynamic coupling is small relative to polarization contribution in this case of high electric field. Therefore, results show that the hydrodynamic coupling effect is a determining factor for RELD at low electric field and becomes a factor that slightly modules RELD curves without changing its shape for high electric field. Figure 4

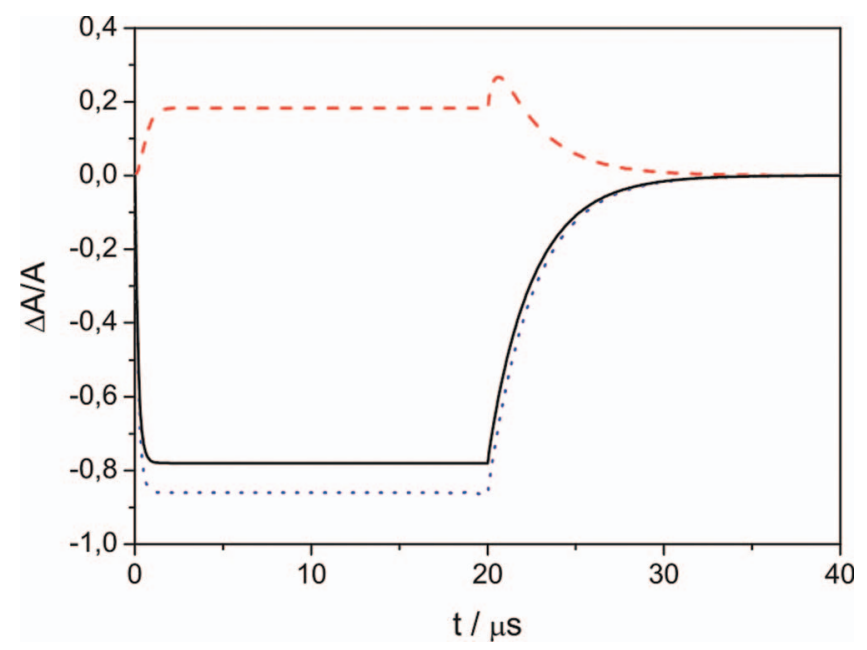

FIG. 4. RELD curve and its decomposition in individual contributions for an electric field strength equal to $60 \mathrm{kV} / \mathrm{cm}$. Solid black line: orientation due polarization and hydrodynamic coupling combined, Dashed red line: contribution owned to hydrodynamic coupling, Dotted blue line: contribution due to polarization.

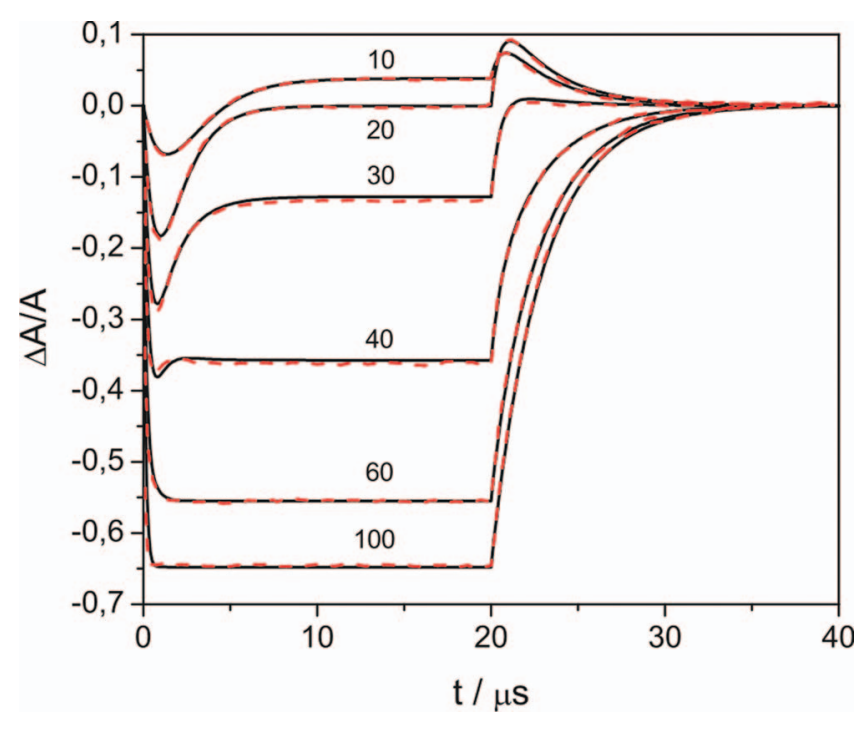

FIG. 5. RELD curves for BRM solution with the same parameters used by Porschke and Antosiewicz ${ }^{4}$ at different electric field strengths given in $\mathrm{kV} / \mathrm{cm}$. Solid black line: values obtained solving Fokker-Planck equation with MDF, Dashed red line: values obtained by Porschke and Antosiewicz ${ }^{4}$ with Brownian dynamic simulation.

shows an overshoot in the hydrodynamic contribution curve (dashed red line) when the electric field is set-off. This overshoot is explained as follows: for only orientation by hydrodynamic coupling, the probability density of finding a molecule in a given orientation range at $t=20 \mu \mathrm{s}$ is greater when the plane $X^{\prime} Z^{\prime}$ of the body coordinate system is aligned in the direction parallel to the electric field. When the electric field is removed, the molecule diffusion around its $Z^{\prime}$ axis is faster than those around the $X^{\prime}$ and $Y^{\prime}$ axes. Therefore, during the first moments after removing the electric field the molecule orientation is such that the absorbance $A_{/ /}$grows with respect to the absorbance $A_{\perp}$, and then also grows the RELD. This behaviour also occurs to $\mathrm{E}=2 \mathrm{kV} / \mathrm{cm}$ but it is not strong enough to see it for this electric field strength.

On the other hand, Figure 4 shows that the different contributions to RELD are clearly not additive. Only in the limit of low electric field the RELD curves approximate to the sum of individual contributions, as can be seen for the case $\mathrm{E}=2 \mathrm{kV} / \mathrm{cm}$ (see Figure 2).

In order to estimate the errors of numeric calculus for high electric fields we contrasted the results of the present approach with those obtained with computational simulation by other authors. Figure 5 shows the RELD curves calculated for a BRM model with the same physical parameters used by Porschke and Antosiewicz, ${ }^{4}$ together with the results obtained for those authors using Brownian dynamic simulation. Both sets of curves are in satisfactory agreement for all electric field strength range (from 10 to $100 \mathrm{kV} / \mathrm{cm}$ ).

\section{CONCLUSIONS}

In this work, previous theoretical developments have been used in combination with numerical procedures to calculate the transient RELD of DNA fragments in aqueous 
solutions. Fokker-Planck equation was numerically solved to describe the orientation of DNA fragments in an electric field using a BRM model characterized by its electric charge, its electric polarizability tensor and its hydrodynamic properties that include the coupling between the translational and rotational movements.

The present work differs with respect to those in the present-day bibliography in two aspects. One of them, arises because this work extends the application range of previous theoretical studies removing the restriction of low electric field; the cost to remove this restriction is the implementation of numerical procedures instead of getting analytical solutions. On the other hand, with respect to those works that use computational simulation this work differs in the use of a completely different technique. We checked that the results of the present numerical calculus are in agreement with those reported in the present-day bibliography.

As the numerical approach here implemented is relatively fast from the point of view of computational time, it is a useful technique to determine physical parameters of DNA fragments in aqueous solution fitting experimental data of transient RELD for different electric field strengths.

\section{ACKNOWLEDGMENTS}

J. P. Umazano is Postdoctoral fellow of Consejo Nacional de Investigaciones Científicas y Técnicas (CONICET) and wishes to thank this Institution.

${ }^{1}$ H. Brenner, Chem. Eng. Sci. 19, 599 (1964).

${ }^{2}$ Y. P. Kalmykov, J. Chem. Phys. 126, 174903 (2007).

${ }^{3}$ D. Porschke, Biophys. Chem. 49, 127 (1994).

${ }^{4}$ D. Porschke and M. Antosiewicz, J. Phys. Chem. 109, 1034 (2005).

${ }^{5}$ M. Antosiewicz and D. Porschke, J. Phys. Chem. 113, 13988 (2009).

${ }^{6}$ W. A. Wegener, R. M. Dowben, and V. J. Koester, J. Chem. Phys. 70, 622 (1979).

${ }^{7}$ W. A. Wegener, J. Chem. Phys. 84, 5989 (1986).

${ }^{8}$ W. A. Wegener, J. Chem. Phys. 84, 6005 (1986).

${ }^{9}$ J. A. Bertolotto, G. B. Roston, M. E. Ascheri, and M. G. Campo, Physica A. 327, 185 (2003).

${ }^{10}$ J. A. Bertolotto, G. B. Roston, and M. E. Ascheri, Prog. Colloid Polym. Sci. 128, 25 (2004).

${ }^{11}$ J. A. Bertolotto, G. B. Roston, G. M. Corral, and M. E. Ascheri, AIP Conf. Proc. 913, 151 (2007).

${ }^{12}$ J. A. Bertolotto, Physica A, 373, 29 (2007).

${ }^{13}$ J. A. Bertolotto, G. M. Corral, E. M. Farias de La Torre, and G. B. Roston, J. Phys.: Condens. Matter 22, 494101 (2010).

${ }^{14}$ J. P. Umazano and J. A. Bertolotto, J. Chem. Phys. 134, 125107 (2011).

${ }^{15}$ S. Mazur and S. A. Allison, J. Phys. Chem. B 101, 2244 (1997).

${ }^{16}$ L. Collatz, The Numerical Treatment of Differential Equations (SpringerVerlag, Berlin, 1966).

${ }^{17}$ J. P. Umazano and J. A. Bertolotto, Mec. Comput. 31, 3397 (2012), http://www.cimec.org.ar/ojs/index.php/mc/article/view/4270. 